\title{
On the Topology of Certain Algebraic Threefold Loci
}

\author{
By J. A. Tond, University of Manchester. \\ (Received 11th March, 1935. Read 4th May, 1935. \\ Received in revised form 8th May, 1935.)
}

\section{Introduction.}

The study of the topological properties of algebraic surfaces, considered as continua of four real dimensions, has thrown much light on the theory of the birational invariants of such loci ${ }^{1}$. The results obtained for surfaces have been generalised to varieties of higher dimension by Hodge ${ }^{2}$, and, particularly, by Lefschetz ${ }^{3}$. Apart from this, little seems to be known about the general topological properties of algebraic loci of three (or more) dimensions, the detailed study of which seems to present considerable difficulty. In particular, apart from the general theorems of Lefschetz, nothing seems to be known about the cycles of three dimensions of an algebraic $V_{3}$. The object of the present paper is to study these cycles on certain quite special $V_{3}$, in the hope that some insight may be gained into the general theory.

In $\S 1$ we recall a theorem which enables the three-dimensional Betti number of an algebraic $V_{3}$ to be calculated in terms of other characters. In $\S 2$ we examine the homology characters of a fairly extensive class of rational $V_{3}$, and describe the nature of the 3-cycles on them. The subsequent sections are devoted to a study of the general cubic primal in [4]. We show that any 3-cycle of this locus is homologous to a 3-cycle lying on a certain algebraic ruled surface on the variety, and may in fact be obtained by the variation of a straight line. This result has at least a negative interest, for if the locus contained non-algebraic 3 -cycles we should be able to deduce surfaces.

1 See Lefschetz, 8, 9; Zariski, 15, for an account of the topology of algebraic

2 Hodge, 6, 7.

${ }^{3}$ Lefschetz, 8, Chap. 5. 
its irrationality; as it is, the matter is still left in doubt. The work may also be of interest as being an application in detail of the general procedure outlined by Lefschetz.

$\S 1$. We consider an algebraic locus $V_{3}$ of three dimensions, irreducible and free from singularities. We denote by $R_{i},(i=1,2, \ldots, 6)$ the $i$-dimensional Betti number ${ }^{1} ; R_{i}=R_{6-i}, R_{0}=1$. We consider on $V_{3}$ a linear system ( $\infty^{3}$ at least) of algebraic surfaces $F$ without singularities, such that the general curve $C$ of intersection of two surfaces $F$ is irreducible. A pencil of surfaces of the system, whose base-curve is $C$, will contain a certain number $n$ of surfaces with a double point. It is known ${ }^{2}$ that

$$
R_{3}=n+2\left(R_{1}+R_{2}\right)-2 r_{2}-r_{1}
$$

where $r_{2}$ is the two-dimensional Betti number of $F$, and $r_{1}$ the onedimensional Betti number of $C$; if $p$ is the genus of $C, r_{1}=2 p$.

\section{§2. The Betti numbers of certain rational varieties.}

Let $C_{p}^{\nu}$ be an irreducible curve of order $\nu$ and genus $p$, without singularities, lying in ordinary space. Consider the linear system $|\Sigma|$ of surfaces of order $m$ which pass through $C_{p}^{\nu}, m$ being chosen so large that $|\Sigma|$ is free from fundamental curves or surfaces. Then $|\Sigma|$ maps the prime sections of a rational locus $V$ in higher space: which for $m$ sufficiently large is free from singularities.

The correspondence between $V$ and the space $S$ containing $|\Sigma|$ is $(1,1)$ except for the points of $V$ mapping the neighbourhoods of points of the base curve $C_{p}^{\nu}$ of $|\Sigma|$. These points on $V$ lie on a ruled surface $R$ whose genus $p$ is the same as that of $C_{p}^{v}$, the neighbourhood of any point on $C_{p}^{\nu}$ corresponding to a line on $R$.

Since $V$ and its prime sections are regular, $R_{1}(V)=0$. Since $V$ is rational it contains a linear system of rational surfaces, corresponding to the planes of $S$. Any 2-cycle on such a surface is

${ }^{1}$ For the fundamental theorems of topology used in the sequel we refer to Lefschetz, 10.

2 Lefschetz, 8, 94. (Theorem XI). 
algebraic, and hence any 4-cycle of $V$ is algebraic ${ }^{1}$. But a base for algebraic surfaces on $V$ is manifestly formed by the prime section of $V$ and the scroll $R$. Hence $R_{4}(V)=2$, and hence by a known duality theorem ${ }^{2}, R_{2}(V)=2$.

To calculate $R_{3}$ we use the formula (1), the system $|F|$ on $V$ being that represented by the planes of $S . F$ is then a rational surface mapped on a plane by curves of order $m$ with $\nu$ base-points, and $C$ is a rational curve. Hence $r_{2}=\nu+1, r_{1}=0$. A surface $F$ will have a node if, and only if, two of the base points in the plane it represents coincide, since $|\Sigma|$ has no fundamental curves. Hence the number $n$ of such surfaces is equal to the rank of $C_{p}^{v}$, i.e. $2 \nu+2 p-2$. Hence (1) gives

$$
\begin{aligned}
R_{3} & =(2 \nu+2 p-2)+2(0+2)-2(\nu+1)-0 \\
& =2 p .
\end{aligned}
$$

We shall now show that the $2 p$ independent 3-cycles on $V$ are precisely the $2 p 3$-cycles on the scroll $R$ which is transformed into the neighbourhood of $C_{p}^{\nu}$. To do this we require a formula connecting $R_{3}(V)$ with the characters of the complexes $R, V-R$. We have, in fact s, $^{3}$

$$
R_{3}(V)=r_{3}(V-R)+R_{3}(R)-s_{3}(R)+t_{3}(R),
$$

where $r_{3}(V-R)$ denotes the number of 3-cycles on $V-R$ which are independent mod. $R, s_{3}(R)$ is the number of 3-cycles on $R$ which bound on $V$, and $t_{3}(R)$ is the number of relative 3-cycles on $V-R$, independent of the $r_{3}(V-R)$ absolute cycles, whose boundaries are homologous to zero on $R$. In the present case $t_{3}(R)$ is zero ${ }^{4}$, and since $V-R$ is homeomorphic ${ }^{5}$ to $S-C_{p}^{v}$,

$$
r_{3}(V-R)=r_{3}\left(S-C_{p}^{\nu}\right)=0 .
$$

Hence, as $R_{3}(V)=R_{3}(R)=2 p, s_{3}(R)=0$, and the $2 p$ 3-cycles of $R$ are independent on $V$.

${ }^{1}$ See Lefschetz, 8, 103. The surfaces mapping the planes of $S$ may be used instead of the prime sections of $V$ itself.

2 Lefschetz, 10, 140.

3 Lefschetz, 10, 150 ; equations (21) and (22).

${ }^{4}$ Lefschetz, 10, 153 ; Theorem I.

${ }^{5}$ Lefschetz, 11, 100. 
The same method clearly extends to the case when the basecurve of $|\Sigma|$ consists of several totally disconnected curves, the system still being free from fundamental elements. If there are $i$ such curves, of genera $p_{1}, \ldots, p_{i}$, it is easily seen that $R_{2}=i+1$; $R_{3}=2 \Sigma p_{i}$.

If fundamental curves and surfaces exist for $|\Sigma|$ the problem becomes more complicated, and the $V_{3}$ may have singularities. The results obtained above exhibit clearly, however, the relative nature of the number $R_{3}$ considered as a birational invariant of $V_{3}$. In general, we may say that the behaviour of $R_{3}$ under birational transformation of $V_{3}$ depends on the genera of the fundamental curves of the transformation, and probably on other factors besides.

The special cases considered here include a number of wellknown loci, notably most of those with rational or elliptic curve sections.

\$5. The Betti numbers and vanishing cycles of the cubic variety in [4].

We consider the general cubic variety $V$ in space of four dimensions, and denote by $|F|$ the system of prime sections of $V$. We recall ${ }^{1}$ that $V$ is the locus of a double infinity of lines of which six pass through each point of the locus, forming an irreducible system whose Grassmannian locus is a surface $\phi$ of irregularity 5 , and hence having $R_{1}(\phi)=10$. Any algebraic surface on $V$ is its complete intersection with another primal'2.

Since the general prime section is a rational surface, any 2-cycle on it is algebraic. Hence every 4-cycle of $V$ is algebraic ${ }^{3}$. Thus, since $V$ only contains complete intersections, any $\Gamma_{4}$ on $V \sim t F$, and $R_{2}=R_{4}=1$. A corollary is that any two lines on $V$ are homologous on $V$, a result otherwise evident, since they correspond to two points of $\phi$, and a chain on $\phi$ joining these defines a continuous deformation of one of the lines into the other on $V$.

Since $V$ is of class 24 and $R_{1}=0$, it follows from (1) that $R_{3}=10$, since $r_{2}(F)=7$ and $r_{1}(C)=2$. Thus there are ten independent 3-cycles on $V$.

${ }^{1}$ See, e.g. Baker, 1, vol. VI, 294 ; Segre, 13, 947. The result is originally due to Fano, 5.

a Fano, 4.

${ }^{3}$ See Lefschetz, 8, 103. 
We now consider a pencil $\left\{F_{u}\right\}$ of $|F|$ whose base is an irreducible curve $C$. For each value of $u$ we have a definite surface $F_{u}$ of the pencil, and for twenty-four values $u_{\mu}$ of $u$ the corresponding surface $F_{\mu}$ has a node. In the plane of the complex variable $u$ we mark the points $u_{\mu}$, and a fixed point $u_{0}$ corresponding to some fixed surface $F_{0}$ of the pencil; and join $u_{0}$ to each point $u_{\mu}$ by a set of nonintersecting arcs. If the plane be cut open along these arcs it becomes $^{1}$ an open 2 -cell $E_{2}$. We can now follow out the variation of any locus on $F_{u}$ as $u$ moves on $E_{2}$.

It is proved by Lefschetz that with each critical value $u_{\mu}$ of $u$ there is associated a definite 2-cycle $\delta_{\mu}$ of $F_{0}$ with the following properties:

(I). If $u$ describes a path on $E_{2}$ from $u_{0}$ to $u_{\mu}$ then the cycle obtained by the variation of $\delta_{\mu}$ vanishes (reduces to a point) when $u=u_{\mu}$.

(II). If $u$ describes a closed path on $E_{2}$ surrounding $u_{\mu}$ and no other critical point, then the initial and final positions $\Gamma_{2}, \Gamma_{2}^{\prime}$, of any 2-cycle on $F_{0}$ are connected by the homology

$$
\Gamma_{2}{ }^{\prime} \sim \Gamma_{2}+\left(\Gamma_{2} \cdot \delta_{\mu}\right) \delta_{\mu},
$$

where $\left(\Gamma_{2} \cdot \delta_{\mu}\right)$ is the usual Kronecker index. Actually Lefschetz gives the coefficient of $\delta_{\mu}$ with a negative sign, but this seems to be a misprint traceable to one in the first equation on p. 92.

In this case we can actually state what the vanishing cycles $\delta_{\mu}$ are. As $u$ tends to $u_{\mu}, F_{u}$ tends to the surface $F_{\mu}$ having a node. On $F_{\mu}$ six lines pass through the node, which arise from the coincidence of two rows of a double-six lying on $F_{u}$. Denoting the lines on $F_{0}$, in the usual Schläfli notation ${ }^{2}$, by $a_{i}, b_{i}, c_{i j}\left(i, j,=1, \ldots, 6 ; c_{i j}=c_{j i}\right)$, so that a typical double-six is

$$
\begin{array}{lll}
a_{1}, & a_{2}, \ldots, a_{6}, \\
b_{1}, & b_{2}, \ldots, b_{6},
\end{array}
$$

1 The procedure is due to Lefschetz, 8, 91. A more detailed account of the construction is given by Zariski, 15, 105, for the case of surfaces; and this account applies to the present case with only a few verbal changes.

2 For the notation, and general properties of the lines on a cubic surface, we refer to Baker, 1, vol. III, Chap. IV. 
this means that as $u$ tends to $u_{\mu}$ the lines $a_{i}$ (or rather the lines on $F_{u}$ obtained by continuous variation from them) tend to coincidence with $b_{i}$, so that as $u$ describes a closed path round $u_{\mu}$ enclosing no other singular point the effect on $F_{0}$ is to interchange the lines $a_{i}, b_{i}$, and leave the lines $c_{i j}$ unaltered. Thus we can take $a_{i}-b_{i}$ to be the cycle $\delta_{\mu}$ which vanishes at $u_{\mu}$, the suffix $i$ being immaterial since the six possible cycles are all homologous on $F_{0}$. A base for 2-cycles on $F_{0}$ is composed of a plane section $C$ and the lines $a_{i}$. If then

$$
\Gamma_{2} \sim t C+\Sigma t_{i} a_{i} .
$$

is a 2 -cycle on $F_{0}$, then when $u$ describes a path round $u_{\mu}, \Gamma_{2}$ becomes $\Gamma_{2}{ }^{\prime}$, where

$$
\begin{aligned}
\Gamma_{2}{ }^{\prime} & \sim t C+\Sigma t_{i} b_{i} \\
& \sim \Gamma_{2}-\left(\Sigma t_{i}\right) \delta_{\mu},
\end{aligned}
$$

and since $\left(C, \delta_{\mu}\right)=0$ and $\left(a_{i}, \delta_{\mu}\right)=-1$,

$$
\Gamma_{2}^{\prime} \sim \Gamma_{2}+\left(\Gamma_{2} \cdot \delta_{\mu}\right) \delta_{\mu},
$$

in accordance with (2). Also, $\left(\delta_{\mu}, \delta_{\mu}\right)=-2$, which shows that $\delta_{\mu}$ is changed in sign as $u$ turns round $u_{\mu}$.

$\S 4^{1}$. If now $u$ describes any closed contour on $E_{2}$, the lines of $F_{0}$ undergo a certain permutation, and the aggregate of all such permutations forms a group $G_{1}$ which either coincides with the group $G$, of order 51840, of all permutations of the lines which preserve their incidence relations, or forms a proper sub-group of $G$. We shall show that in fact $G_{1} \equiv G$.

Any closed path on $E_{2}$ starting from $u_{0}$ can be deformed into the sum of a finite number of loops each surrounding a single one of the points $u_{\mu}$. Thus $G_{1}$ is generated by the permutations effected when $u$ describes each of these loops. Each of these permutations is of the type described in the previous section, interchanging the two rows of one of the double-sixes on $F_{0}$ and leaving the fifteen lines which do not belong to the double-six unaltered.

It is well known ${ }^{2}$ that $G$ can be represented as a group of

1 The results in this section are not used in the rest of the paper, but are inserted for their intrinsic interest.

2 See Schoute, 12 ; Coxeter, 2, 165 ; Todd, 14. 
congruent transformations in Euclidean space of six dimensions, leaving invariant a certain polytope with thirty-six pairs of opposite vertices (in correspondence with the double-sixes on $F_{0}$ ), having also thirty-six primes of twofold symmetry which bisect the diagonals joining pairs of opposite vertices at right angles. The operation of $G$ which interchanges the two rows of a double-six is represented by the reflection in the corresponding prime, and the whole group is simply isomorphic with the group of rotations and reflections of the polytope. Consequently $G_{1}$ is generated by reflections in a certain number of the primes of symmetry of the polytope, and so is included in the table of such groups given by Coxeter ${ }^{1}$.

We next show that $G_{1}$ acts transitively on the lines of $F_{0}$; to do which it is sufficient to show that if $l_{1}$ and $l_{2}$ are any two lines of $F_{0}$ there is an operation of $G_{1}$ which interchanges $l_{1}$ and $l_{2}$. Let $L_{1}$ and $L_{2}$ be the points on the Grassmannian $\phi$ of the lines on $V$ which correspond to $l_{1}$ and $l_{2}$. The lines of $V$ which occur on the surfaces of the pencil $\left\{F_{u}\right\}$ all meet the base curve $C$ of the pencil, and conversely any line of $V$ which meets the plane of $C$ meets $C$ and lies on just one of the surfaces $\left\{F_{u}\right\}$. These lines thus belong to a (special) linear complex and are mapped by the points of a curve $\omega$ on $\phi$ (actually a prime section of $\phi$ ), which is irreducible and which passes through $L_{1}$ and $L_{2}$. This curve $\omega$ (or rather the corresponding Riemann surface) is in $(27,1)$ correspondence with the complex plane on which $u$ is represented, since each $F_{u}$ contains 27 lines. Thus any arc on $\omega$ joining $L_{1}$ and $L_{2}$ corresponds to an arc in the $u$-plane, which is a cycle since $L_{1}$ and $L_{2}$ both correspond to the same point $u_{0}$. We can choose this arc on $\omega$ so that it does not meet any of the arcs whose points correspond to points of the $u$-plane on the cuts $u_{0} u_{\mu}$. Then the cycle in the $u$-plane lies on $E_{2}$, and, as $u$ describes the cycle, $l_{1}$ is carried into $l_{2}$. This proves that $G_{1}$ is transitive on the lines of $F_{0}$.

It is now easily seen that $G_{1}$ coincides with $G$. For if not, it is a proper subgroup of $G$ occurring in the table in Coxeter's paper referred to above. All these groups are easily verified to be subgroups of one or other of the three groups denoted by the symbols

$$
\left[3^{2,1,1}\right], \quad\left[3^{4}\right] \times[], \quad[3]^{3} .
$$

1 Coxeter, 3, 480. (Table VI, (iv)). 
The first of these is the subgroup of $G$ keeping fixed a definite line of $F_{0}$, the second leaves a particular double-six invariant, while the third leaves invariant nine lines of $F_{0}$ which belong to a Steiner trihedral. Thus all the proper subgroups of $G$ generated by a partial set of the reflections which generate $G$ are intransitive on the lines of $F_{0}$. Hence, since $G_{1}$ is transitive, $G_{1} \equiv G$.

\section{§5. The homologies connecting the vanishing cycles.}

The vanishing cycles considered in $\S 3$ are all of the type $l_{1}-l_{2}$, where $l_{1}$ and $l_{2}$ are two non-intersecting lines of $F_{0}$. Since $F_{0}$ contains 216 pairs of non-intersecting lines there are 216 cycles of this form on $F_{0}$, which fall into 36 sets of six homologous cycles corresponding to the 36 double-sixes on $F_{0}$. These sets are themselves connected by homologies, since it is easily seen that only six of these cycles can be linearly independent on $F_{0}$. For we may take a representative of each of the 36 sets of cycles, for instance

$$
a_{1}-a_{i} ; \quad a_{1}-b_{i} ; \quad a_{1}-c_{i j} ; \quad b_{1}-c_{i j} ; a_{i}-a_{j} ;
$$

with the convention $2 \leqq i<j \leqq 6$. The six cycles

$$
a_{1}-a_{3} ; a_{1}-a_{4} ; \quad a_{1}-a_{5} ; \quad a_{1}-a_{6} ; a_{1}-b_{1} ; a_{1}-c_{23}
$$

are independent on $F_{0}$, and the others are expressible in terms of these in virtue of the homologies

$$
\left.\begin{array}{rl}
\left(a_{1}-a_{2}\right) \sim\left(a_{1}-b_{1}\right)+\left(a_{1}-a_{4}\right)+\left(a_{1}-a_{5}\right)+\left(a_{1}-a_{6}\right)-\left(a_{1}-a_{3}\right)-2\left(a_{1}-c_{23}\right) \\
& \sim\left(a_{1}-b_{1}\right)+\left(b_{1}-b_{4}\right)+\left(c_{56}-c_{16}\right)+\left(c_{63}-c_{13}\right) \\
& -\left(c_{36}-c_{16}\right)-\left(a_{2}-c_{13}\right)-\left(c_{56}-b_{4}\right), \\
\left(a_{i}-a_{j}\right) & \sim\left(a_{1}-a_{j}\right)-\left(a_{1}-a_{i}\right), \\
\left(a_{1}-c_{2 k}\right) & \sim\left(a_{1}-c_{23}\right)+\left(a_{1}-a_{3}\right)-\left(a_{1}-a_{k}\right) \\
& \sim\left(a_{1}-c_{23}\right)+\left(c_{32}-c_{12}\right)-\left(c_{k 2}-c_{12}\right), \\
\left(a_{1}-c_{j k}\right) & \sim\left(a_{1}-c_{j 2}\right)+\left(a_{1}-a_{2}\right)-\left(a_{1}-a_{k}\right) \\
& \sim\left(a_{1}-c_{j 2}\right)+\left(c_{2 j}-c_{1 j}\right)-\left(c_{k j}-c_{1 j}\right), \\
\left(b_{1}-c_{j k}\right) & \sim\left(a_{1}-c_{j k}\right)-\left(a_{1}-b_{1}\right) .
\end{array}\right\}
$$

The homologies (3) evidently form a minimal basis for homologies between the vanishing cycles, and the form of these homologies makes it clear that in any such homology we can arrange, by replacing the individual vanishing cycles by others belonging to the same set of six on $F_{0}$, that the actual expressions on the two sides of the homology 
sign are algebraically the same when expressed in terms of the lines $a_{i}, b_{i}, c_{i j}$. Consequently we have the following result. Any homology

$$
\Sigma \lambda_{a} \delta_{a} \sim 0
$$

between the vanishing cycles $\delta_{\alpha}$ of $F_{0}$ is equivalent to a homology

$$
\Sigma \mu_{\beta} \delta_{\beta}{ }^{\prime} \sim 0
$$

in which each of the cycles $\delta_{\beta}{ }^{\prime}$ is a vanishing cycle homologous on $F_{0}$ to one of the cycles $\delta_{a}$, and the algebraic sum of the cycles on the left, expressed in terms of the lines of $F_{0}$, is zero. [Of course it is not necessarily the case that each of the $\lambda_{a}$ cycles $\delta_{a}$ in (4) is replaced by the same cycle $\delta_{\beta}{ }^{\prime}$; an example is the first homology (3), where $2\left(a_{1}-c_{23}\right)$ is replaced by $\left(a_{1}-c_{23}\right)+\left(c_{56}-b_{4}\right)$.]

\section{§6. Determination of the 3-cycles on $V$.}

It is shown by Lefschetz in his book ${ }^{1}$, that for any 3 cycle $\Gamma_{3}$ of $V$ we have the homology $\Gamma_{3} \sim \Sigma \lambda_{\mu} \Delta_{\mu}+M_{3}$, where $\Delta_{\mu}$ is the 3-chain described by $\delta_{\mu}$ as $u$ describes the arc $u_{0} u_{\mu}$, and $M_{3}$ is a 3-chain on $F_{0}$ whose boundary is $-\Sigma \lambda_{\mu} \delta_{\mu}$; and that such a cycle arises from every independent homology of type (4). Now if $\delta_{\mu}{ }^{\prime}$ is one of the other vanishing cycles at $u_{\mu}$, and $\Delta_{\mu}{ }^{\prime}$ its locus as $u$ describes $u_{0} u_{\mu}$, then $\Delta_{\mu}{ }^{\prime}-\Delta_{\mu}$ is a 3-chain bounded by the 2-cycle $\delta_{\mu}{ }^{\prime}-\delta_{\mu}$. Further, $\delta_{\mu}{ }^{\prime}-\delta_{\mu}$ bounds a 3 -chain $N_{3}$ in $F_{0}$ such that $\Delta_{\mu}{ }^{\prime}-\Delta_{\mu}-N_{3}$ is a bounding 3-cycle of $V$. Hence in view of the result of the last paragraph we may suppose that $\Sigma \lambda_{\mu} \delta_{\mu}$ is algebraically zero. Then $M_{3}$ must be a cycle. But any 3-cycle on $F_{0} \sim 0$. Thus every 3-cycle on $V$ is of the form $\Sigma \lambda_{\mu} \Delta_{\mu}$ where $\Sigma \lambda_{\mu} \delta_{\mu}$ is algebraically zero. Since $\delta_{\mu}$ is of the form $l_{1}-l_{2}$, where $l_{1}, l_{2}$ are two lines of $F_{0}$ which are interchanged when $u$ describes a closed path round $u_{\mu}, \Delta_{\mu}$ is the locus of $l_{1}$ as $u$ describes this circuit. Hence every 3-cycle of $V$ may be regarded as the locus of a line of $V$ as $u$ describes a suitable closed path on $E_{2}$; and is therefore reducible to a cycle on the scroll formed by the lines of $V$ which meet $C$. The ten independent cycles accord with the fact that the Grassmannian $\phi$ of the lines of $V$ carries ten independent 1-cycles.

1 Lefschetz, 8, 93. (Theorem VI). 


\section{REFERENCES.}

1. H. F. Baker, Principles of Geometry (Cambridge), III (1923), VI (1933).

2. H. S. M. Coxeter, "The polytopes with regular-prismatic vertex figures," Part 2, Proc. London Math. Soc. (2) 34 (1932) 126-189.

3. H. S. M. Coxeter, "Finite groups generated by reflections, and their subgroups generated by reflections." Proc. Camb. Phil. Soc., 30 (1934) 466-482.

4. G. Fano, "Sulle superficie algebriche contenute in una varietà cubica dello spazio a quattro dimensioni." Atti Acc. Torino, 39 (1904) 597-615.

5. G. Fano, "Sul sistema $\infty^{2}$ di rette contenute in una varietà cubica generale dello spazio a quattro dimensioni." Atti Acc. Torino, 39 (1904) 778-792.

6. W. V. D. Hodge, "On multiple integrals attached to an algebraic variety." Jour. London Math. Soc., 5 (1930) 283-290.

7. W. V. D. Hodge, "Some theorems on abelian integrals associated with an algebraic variety." Proc. Camb. Phil. Soc., 31 (1935) 18-25.

8. S. Lefschetz, L'analysis situs et la géométrie algébrique (Paris, 1924).

9. S. Lefschetz, Géométrie sur les surfaces et les variétés algébriques (Mémorial des Sciences Mathématiques, 40, 1929).

10. S. Lefschetz, Topology (New York, 1930).

11, S. Lefschetz, "Invariance absolue et invariance relative en géométrie algébrique." Recueil Math. Soc. Moscou, 39 (1932) 98-103.

12. P. H. Schoute, "On the relation between the vertices of a definite six-dimensional polytope and the lines of a cubic surface." Proc. Roy. Acad. Sci. (Amsterdam) 13 (1910) 375-383.

13. C. Segre, Mehrdimensionale Räume (Encyk. Math. Wiss., III C 7).

14. J. A. Todd, "Polytopes associated with the general eubic surface." Jour. London Math. Soc. 7 (1932) 200-205.

15. O. Zariski, Algebraic Surfaces (Ergebnisse der Math. III 5, 1935). 